\title{
Kuifi kimün aukantun kimeltuwün meu. La enseñanza del juego mapuche desde las lógicas internas de su cultura Kuifi kimün aukantun kimeltuwün meu. The teaching of the Mapuche game from the internal logic of its culture \\ *, **Carolina Poblete Gálvez, ***Alberto Moreno Doña, ****Eduardo Sandoval Obando, *Esteban Aedo Muñoz

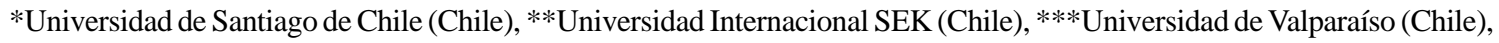 ****Universidad Autónoma de Chile (Chile)
}

\begin{abstract}
Resumen. Este artículo trata la temática específica del juego ancestral mapuche y la enseñanza en el contexto actual de la revitalización cultural de este pueblo indígena en la sociedad chilena. Investigamos el impacto sociocultural de este aprendizaje en el contexto del kimeltuwün para el aporte de la transmisión del conocimiento mapuche en espacios y tiempos educativos de la propia comunidad. Estudio fenomenológico cualitativo, evidenció las narrativas co-construidas en dos comunidades mapuche y escuelas en la región de la Araucanía, Chile. Los resultados revelan el valor, uso del juego y orientaciones metodológicas.
\end{abstract}

Palabras clave: Juego educativo, autóctonos, manifestaciones culturales.

Summary. This article deals with the specific theme of the Mapuche ancestral game and its teaching in the current context of the cultural revitalization of these indigenous people in Chilean society. We investigate the sociocultural impact of this learning in the context of kimeltuwün as a contribution for the transmission of Mapuche knowledge in educational spaces and times within its community. The qualitative phenomenological study highlighted the narratives co-constructed in two Mapuche communities and schools from the Araucanía region, Chile. The results revealed the value of the game, its use, and the methodological orientations.

Keyworks: Educational game, autochthonous, cultural events.

\section{Introducción}

Por mucho tiempo nos han enseñado, desde los referentes clásicos de la cultura occidental, que el juego es recreativo, ocio y parte esencial de la infancia. Autores clásicos como Huizinga (1949), Cagigal (1976) y Moor (1987) señalan que el juego es, dados sus componentes identitarios, generación de cohesión social, sentido de comunidad, que antropológicamente es fundamental en los procesos educativos. Desde la sociología el juego es descrito como un transmisor de conductas y costumbres o como una forma de explicar la vida de las personas en comunidad (Rodríguez, Oliveira y Navarro, 2019). Algunos autores latinoamericanos contemporáneos como Maturana (2003) y Altuve (2009), han dado un giro a las ideas clásicas y han apropiado al juego de su carácter simbólico, situado y emocional. Es decir, el juego que encarna el ser mismo de quien lo juega, revelándose ante él su esencia en este contexto. Desde el giro cultural, enfatizan el concepto de juego en torno a la idea de diversidad como manifestación cultural de los pueblos originarios (Kusch, 1977). La caída de los grandes relatos históricos hegemónicos, dada la crítica hacia las memorias culturales de Abya Yala (en el caso de esta investigación del Wallmapu), perfilan e insisten en el rigor de la resistencia (Hopenhayn, 2005) y de la razón indolente (Santos, 2000) para ir más allá de lo evidente y con ello levantar las emergencias culturales que encierra el juego ancestral, que en el contexto mapuche, permitirían comprender otras forma de configuración social u otras formas de existencia cultural mostrando nuevas aristas, incluso complementarias, de la visión que se tenía del juego en la cultura hegemónica occidental.

El juego encierra un sin número de significados según sea la cultura, el momento histórico o la naturaleza explicati-

Fecha recepción: 07-05-19. Fecha de aceptación: 08-08-19

Carolina Poblete Gálvez

carolina.poblete.g@usach.cl va de su creación. Si bien podrían reproducirse elementos estructurales, no así los contextos socioculturales. El caso de uno de los juegos en esta investigación se refleja lo señalado. El Palin, juego ancestral mapuche se ha asemejado con el Hockey (Garoz, 2002, López, 2011) a pesar de que tienen solo algunos elementos estructurales que los asemejan. Esta comparación podría desvalorizar, en sí mismo, el valor propio del juego ancestral, validando como imaginario solo lo que pertenece a la esfera de la cultura dominante. Esto es lo que refleja el juego mapuche en el contexto de la literatura histórica de la sociedad chilena. En este caso al deporte moderno. Se cuida en esta investigación evitar este tipo de analogías. En este mismo sentido, el contexto de esta investigación es la escuela, lugar donde hemos considerado encontrar formas favorables para el aprendizaje del juego mapuche desde el kimeltuwün, sin embargo experiencias como las planteadas por Rodríguez, Oliveira y Navarro (2019) nos alertan frente a que «los espacios disponibles en la escuela condicionan sobremanera las actividades realizadas por niños y niñas. Campos de fútbol de hierba artificial, pistas de baloncesto, porterías reglamentarias de balonmano o fútbol sala (...) orientan a las nuevas generaciones a la práctica de actividades modernas, novedosas y atractivas a primera vista, en detrimento de las prácticas tradicionales de antaño» (p.29).Es necesario partir de la base que el juego mapuche en este sentido no ha formado nunca parte de la educación chilena de manera concreta.

Por herencia cultural, el conocimiento mapuche se transmite oralmente. Sin embargo, los esfuerzos de quienes han intentado regístralo en forma escrita han contribuido a su pervivencia para las nuevas generaciones, pese a la imposición monocultural de la educación chilena (Quilaqueo \& Torres, 2013) y las políticas asistencialistas de revitalización cultural (Poblete, 2018). Por otra parte, pero en contexto, la lengua originaria de esta cultura, como configuración de una realidad y significante sociocultural, se encuentra en inferioridad y en los márgenes de la lengua dominante (Naguil, 2018). Es importante considerar que cada cultura asigna, 
mediante el lenguaje, formas valóricas de convivencia y relación. En el caso mapuche existen algunos conceptos identificados desde su lengua de origen, a saber. El aukantun es el concepto que define la acción de jugar actualmente y kimeltuwün hace referencia al contexto educativo de aprendizaje o la forma de aprender mapuche. Los juegos ancestrales mapuche son parte del kimün, conocimiento o sabiduría, por lo tanto kimün aukantun es todo conocimiento acerca del jugar. El desafío real de su práctica es descifrar el kimün aukantun para comprender cómo el mapuche se encuentra con su cultura u otras personas encuentran lo mapuche en el juego. Este proceso es imposible sin la comprensión del lenguaje originario en la práctica de estas lúdicas.

\section{Referentes conceptuales}

Los antecedentes y referentes teóricos que sustentan nuestro trabajo investigativo en torno al kiimün aukantun son: Quilaqueo (2006), Quilaqueo y Quintriqueo (2008), Quilaqueo y Quintriqueo (2010) y Duran y Catriquir (2007). Nos alumbran desde las lógicas educativas interculturales bilingües, las que contienen, desde el mapuzugun (lengua originaria mapuche), ideas y conceptos definidos acerca de lo que significa la idea de educarse para el pueblo mapuche. La tabla n¹ explica los conceptos elegidos desde la lógica del kimeltuwün que plantean estos autores y que ha sido utilizada en esta investigación como contexto de análisis.

En relación al contexto educativo mapuche consideramos que los trabajos de Quintriqueo y Matreux (2004); Quilaqueo, Quintriqueo y Cardenas (2005); Quidel (2002), Quilaqueo, Quintriqueo, Torres y Muñoz (2014), nos orientan para comprender las limitaciones del proceso de enseñanza en contexto intercultural. También nos aportan, desde experiencias concretas, a entender cómo los procesos de revitalización cultural mapuche, en la esfera de la educación escolar en Chile, se diluyen en esfuerzos intencionales muy poco contextualizados al proceso histórico del desarraigo del pueblo mapuche. Dicho en términos concretos, la educación chilena posee pocas estrategias de reparación de las consecuencias históricas de usurpación y asimilación cultural a la que ha sido sometida la sociedad mapuche desde hace poco más de un siglo. En la lógica descriptiva de Walsh (2009), la educación intercultural en Chile es de tipo funcional, es decir, le es útil a los discursos políticos educativos, pero carecen de toda crítica en cuanto al significado real que generan quienes son educados en este sentido.

Hemos considerado, también, para esta última idea, los trabajos sobre los contextos sociales e históricos mapuche de Merino y Quilaqueo (2007); Mariman, P.; Caniuqueo, S.; Millalén, J. y Levil, R. (2006) y Paillalef (2003). Estos autores coinciden en que el proceso de revitalización de la cultura mapuche va de la mano con los procesos de autonomía al interior de dicha cultura, la que desde luego choca con las imposiciones estatales de la nación hegemónica chilena en la que circulan estos saberes. Desde los trabajos críticos de Torres (2001) y Calvo (2008) podemos argumentar que los procesos escolares elaborados en los tiempos actuales, están referidos, principalmente, a la problemática de la educación de mercado que posee Chile y que permea todas las esferas de las políticas educativas, así como las lógicas es- tructurales y monoculturales del currículum. Y que en este mismo sentido, prácticas culturales como el juego, que carecen de necesidad de consumo directo del mercado deportivo y recreativo, pueden verse vinculadas fuertemente a espacios de resistencia, transformación y crítica social (Gonçalves, Aparecida y Rodríguez; 2018).

Estas referencias han dado forma a esta investigación y han permitido la selección de conceptos claves que dirigen nuestra propuesta de análisis, la cual se centra en comprender las lógicas de enseñanza del juego mapuche desde su propia forma de educación llamada kimeltuwün. Los conceptos elegidos, descritos en la tabla $\mathrm{n}^{0} 1$, son el contexto de análisis en los focos grupales realizados en esta investigación. Si bien estos conceptos son la base de análisis, también es posible identificar otros que nutren el proceso investigativo, pues somos conscientes que en el proceso de comprensión de lo mapuche, y en particular la comprensión de la dimensión del juego, existen aún vacíos conceptuales que fueron desprendiéndose del trabajo de investigación.

Desde las referencias teórico descriptivas del juego mapuche, es posible identificarlo en Manquilef (1914); López (2011) López (20013), el primero mapuche, describe los tipos de juego existentes en la zona de Metrenco a principios de siglo XX, el segundo autor, describe etnográficamente la situación de algunos juegos a fines de este mismo siglo. Estas referencias nos permiten comprender las lógicas estructurales de los 6 juegos que elegimos, para luego llevarlas a la enseñanza del juego desde el kimeltuwün. Los juegos elegidos desde la literatura fueron: Palin, Linao, Awarkuzen, Kechucawe, Maumillan y Komikan

\begin{tabular}{ll}
$\begin{array}{l}\text { Tabla no1. } \\
\text { Conceptos del Kimeltuwün }\end{array}$ \\
$\begin{array}{l}\text { Concepto } \\
\text { Criterios teóricos }\end{array}$ & \multicolumn{1}{c}{$\begin{array}{c}\text { Descripción según autores. } \\
\text { Quilaqueo (2006), Quilaqueo y Quintriqueo (2008) y Quilaqueo y } \\
\text { Quintriqueo (2010) }\end{array}$} \\
\hline Gülam & $\begin{array}{l}\text { Es una estrategia utilizada para la enseñanza de valores y actitudes respecto } \\
\text { del conocimiento social, la naturaleza, la creación artístico-tecnológica y lo } \\
\text { espiritual-simbólico de acuerdo con el ideal de az (rasgos/ } \\
\text { comportamientos/ característica) mapuche }\end{array}$ \\
\hline Inatuzugun & $\begin{array}{l}\text { Corresponde al ejercicio de búsqueda en las raíces familiares sobre el ser } \\
\text { mapuche o az de la persona. }\end{array}$ \\
\hline Azmuwün & $\begin{array}{l}\text { Valor actitudinal educativo de respeto mutuo entre las personas y entre las } \\
\text { personas y el medio natural, para una mejor armonía y desarrollo humano. }\end{array}$ \\
\hline Mañummuwün & $\begin{array}{l}\text { Valor actitudinal educativo, se refiere a la forma de agradecimiento de la } \\
\text { persona con las otras, medio natural y el mundo espiritual. }\end{array}$ \\
\hline Yamuwün & $\begin{array}{l}\text { Valor actitudinal educativo, se entiende como el aprecio y la valoración de } \\
\text { las personas, el respeto a seres naturales y sobrenaturales reconocidos como } \\
\text { positivos para el desarrollo de las personas y su entorno. }\end{array}$ \\
\hline Zapin & $\begin{array}{l}\text { Corresponde a la forma de ser cuidadoso/a o enseñanza de actitudes para } \\
\text { lograr cultivar un saber y construir un conocimiento o cultivarse. }\end{array}$ \\
\hline
\end{tabular}

\section{Metodología}

Esta investigación se centra en el paradigma cualitativo. Es un estudio fenomenológico-interpretativo debido a que nuestro objetivo general es la comprensión educativo-cultural que implica la práctica del juego ancestral Mapuche bajo su propia lógica a través de la técnica del Foco grupal. Iniciamos con preguntas orientadoras: ¿Cuál es el impacto sociocultural del kuifi kimün aukantün desde el kimeltuwün para la transmisión del conocimiento mapuche en espacios y tiempos educativos de la propia comunidad?, ¿Cuáles son las percepciones acerca del aprendizaje de la práctica de kuifi mapuche aukantun en los niños y las niñas de cuatro contextos rurales de educación de la comuna de Padre Las Casas, Temuco, Chile? Y ¿cómo entiende la comunidad mapuche de Padre Las Casas los conceptos de Zapin, Inaltuzugun, 
Gülam, Yamuwün, Mañummawün, y Azmuwün para el contexto del kimletuwün?

En base a estos primeros cuestionamientos definimos los objetivos de investigación. Como objetivo general: comprender el impacto sociocultural del aprendizaje del kuifi kimün aukantun bajo el contexto del kimeltuwün para el aporte de la transición del conocimiento mapuche en espacios y tiempos educativos de la propia comunidad. Nuestros objetivos específicos fueron los siguientes:

1. Describir, contextualizadamente, el conocimiento ancestral del juego mapuche desde en las lógicas del kimeltuwün, considerando: zapin, gülam, inaltuwün yamuwün, azmawün y mañummawün a partir de los aportes de dos comunidades aledañas a las escuelas rurales.

2. Significar las percepciones acerca del aprendizaje de la práctica de kuifi mapuche aukantun en las lógicas del kimeltuwün para niños y niñas en cuatro escuelas rurales de la comuna de Padre Las Casas.

Hemos utilizado la descripción, relación e interpretación de los hallazgos alcanzados durante el proceso investigativo. Se pidió a las comunidades participantes que aportaran, desde su entendimiento, al diseño didáctico de enseñanza de los juegos en la lógica del kimeltuwün. En el caso de los grupos focales para niños y niñas se recogió las opiniones de su experiencia lúdica en los talleres diseñados bajo la lógica del kimeltuwün, en base al cruce de información de los conceptos del kimeltuwün y las posibilidades de materialización en el juego mapuche. Este diseño metodológico de enseñanza (Tabla $n^{\circ} 2$ ) fue propuesto para indagar en las actitudes y reacciones de los niños y niñas frente al tema de la enseñanza del juego mapuche en el contexto del kimetuwün. Las preguntas fueron respondidas en la interacción del grupo en una jornada de reunión-desayuno o trawün (en lengua mapuche) para el caso de 2 comunidades y, en el caso de los niños y niñas realizamos 4 foco grupal al finalizar cada experiencia lúdica a través del nütramkan (forma de dialogar mapuche), una dinámica en que los participantes se sienten cómodos y libres de hablar y comentar sus opiniones. Orientamos la conversación hacia la participación en cuanto a, describir los detalles minuciosamente, las experiencias complejas y razonamientos que dirigen sus acciones, creencias, percepciones y actitudes (Bertoldi, Fiorito \& Álvarez, 2006; Morse, 2005; Edwards \& Stokoe, 2004).

Tabla n ${ }^{\circ} 2$.

Propuesta metodológica llevada a cabo con el cruce contextual de conceptos.

Propuesta metodológica de viculación del mapuche aukantun con conceptos del \begin{tabular}{cc}
\multicolumn{2}{c}{ KIMELTUWÜN } \\
\hline KIMELTUWÜN / AUKNATUN & Contexto descrito \\
\hline Se les pide a los niños y niñas que establezcan conversaciones y
\end{tabular} Gülam Awarkuzen diálogos diversos al momento de jugar. Quienes enseñamos

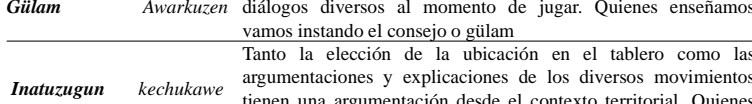

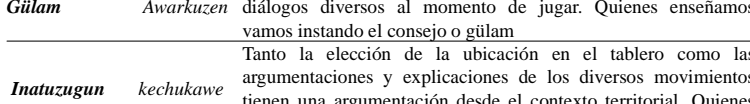
Inatuzugun kechukawe $\begin{aligned} & \text { argumentaciones y explicaciones de los diversos movimientos } \\ & \text { tienen una argumentación desde el contexto territorial. Quienes }\end{aligned}$ enseñamos vamos aportando datos de estos territorios. Narramos epew (fabulas) para explicar el juego, luego hacemo nütramkan para hablar de las características y las formas de ser de
los protagonistas del juego. los protagonistas del juego. Iniciamos el juego recordando el rol e importancia de este juego
para la cultura mapuche y de las cosas que podemos aprender. para la cultura mapuche y de las cosas que podemos aprende. figura del "kon" (par del juego) al que hay que atender y cirdr en las lógicas de este juego. eoordinamos en acuerdo las formas de resguardar la seguridad de entre los pares, imitando sonidos y palabras en mapuzugun. Realizamos nutramkan en torno al concepto de respeto y las formas de ser respetuosos/as y las consecuencias vista en el juego ste juego altamente enérgico, se debe practicar bajo el acuer

en la permisividad del contacto físico y el cuidado entre pares $d$

$\begin{array}{lll}\text { Zapin Linao } & \begin{array}{l}\text { en la permisividad del contacto físico y el cuidado entre pares de } \\ \text { juego. Al igual que en los anteriores el nutramkan es necesario }\end{array}\end{array}$

$\begin{array}{lll}\text { Zapin Linao } & \begin{array}{l}\text { en la permisividad del contacto físico y el cuidado entre pares de } \\ \text { juego. Al igual que en los anteriores el nutramkan es necesario } \\ \text { antes y después de la práctica }\end{array}\end{array}$ Para ver la metodología revisar: https://www.youtube.com/watch?v=HrHsvADx5Q0
En el grupo focal 1-2 participaron 30 adultos y adultas de la comunidad de Padre Las Casas que cumplían con los siguientes criterios de inclusión: Pertenencia indígena mapuche, pertenecer a la comunidad invitada, deseablemente apoderados de los establecimientos participantes de la investigación, mayores de edad, mínimo de participantes 10 personas. Los grupos focales 3-4-5-6 se realizaron con niños/as que asisten a las escuelas rurales (cuatro escuelas) de la comunidad de Padre Las Casas. Estos participantes conversaron acerca de sus experiencias lúdicas. Los niños/ as que participaron debieron cumplir con los siguientes criterios de inclusión: cantidad total de participantes del taller de juegos, niveles de cuarto a sexto, todos los géneros. Todos los participantes en este trabajo de investigación realizaron su participación de manera voluntaria bajo consentimiento y asentimientos informados según el caso y protocolo ético.

El análisis de los datos se llevó a cabo bajo la lógica de fragmentación y articulación de la «Grounded Theory» (Strauss \& Corbin, 2002). Primero se redujeron los datos desde la simplificación, resumen y selección de la información. Para Strauss y Corbin (2002:110), «éste sería el proceso analítico por medio del cual se identifican los conceptos y se descubren en los datos sus propiedades y dimensiones». A través de una lectura analítica de los datos se seleccionaron las unidades de significado previas en base a «criterios temáticos» (Rodríguez; Gil y García, 1999, p. 207). El proceso seguido fue fundamentalmente inductivo (Miles \& Huberman, 1994), aunque lógicamente existen una serie de temáticas y referencias teóricas descritas en el marco de referencia de este trabajo, desde los cuales se orientan los análisis y que son ejes centrales de la investigación. Para facilitar el proceso de análisis se utilizó en software de análisis cualitativo Nvivo12.

Posteriormente se realizó la síntesis y agrupamiento de los criterios temáticos, lo que nos posibilitó juntar varias unidades y códigos bajo un mismo tópico o concepto teórico (Rodríguez; Gil \& García 1999). Esta labor de síntesis estuvo orientada a una recreación de lenguaje, que corresponde en la literatura a la transformación de los datos (Strauss \& Corbin, 2002). En el procedimiento de representación de los hallazgos optamos por las representaciones gráficas o imágenes visuales de la relación entre conceptos (Strauss y Corbin, 2002). Finalmente, se materializó la obtención de resultados y conclusiones, fundamentalmente en la contextualización y constatación de los hallazgos alcanzados con los estudios similares en la temática de la educación para el contexto mapuche. Los nombres utilizados para la ejemplificación de los hallazgos son ficticios para el resguardo de la identidad de las personas participantes.

\section{Resultados}

En la comprensión del impacto sociocultural del aprendizaje del kuifi kimun aukantun bajo el contexto del kimeltuwun para el aporte de la transición del conocimiento mapuche en espacios y tiempos educativos de la propia comunidad, objetivo general de nuestro trabajo investigativo, hemos podido llegar realizar un trabajo de análisis de las narrativas coconstruidas por niños(as) y adultos de esta comunidad. Los 
grupos focales donde ellos participaron nos han permitido ordenar y sistematizar las grandes ideas desde las que la comunidad escolar y mapuche de Padre Las Casas entienden el impacto del juego en sus vidas en general y en la escuela en particular.

En la presentación de este hallazgos seguiremos una lógica caracterizada por recorrer un camino desde lo más general de las narrativas, enfatizando las temáticas con mayor presencia en los discursos, hacia lo más particular; aspecto éste último que nos permitirá profundizar lo dicho por los sujetos participantes.

Hemos creído relevante comenzar la presentación de los hallazgos con una nube de palabras (Figura 1) que representa los conceptos, ideas y temáticas más fácilmente detectables en los discursos analizados en la dos comunidades mapuche.

Al visualizar esta nube de palabras, llama poderosamente la atención la relevancia que para las comunidades tiene la idea de persona. Es alrededor de esta persona, no entendida como un ser individual (en esto profundizaremos más adelante) sino como un/a sujeto constituido por y constituyente de una comunidad, el cual posee la distinción de ser mapuche, es decir se hace hincapié en la forma de entender el ser persona desde lo que para cultura mapuche se entiende esta idea. Este ser persona implica los valores encarnados en los juegos que presentamos a los niños y niñas y es por tanto esta idea del aprender a ser persona mapuche un elemento articulador del valor e impacto del juego y la ludicidad propia en la cultura mapuche en la opinión de las comunidades mapuche a las que consultamos.

Si queremos ser un poco más exhaustivos en relación a las grandes temáticas presentes en los discursos producidos los niños y niñas esta vez, en relación al valor del juego, es interesante visualizar que la gráfica refuerza la idea del ser persona mapuche en el juego (Figura 2) pero además desde
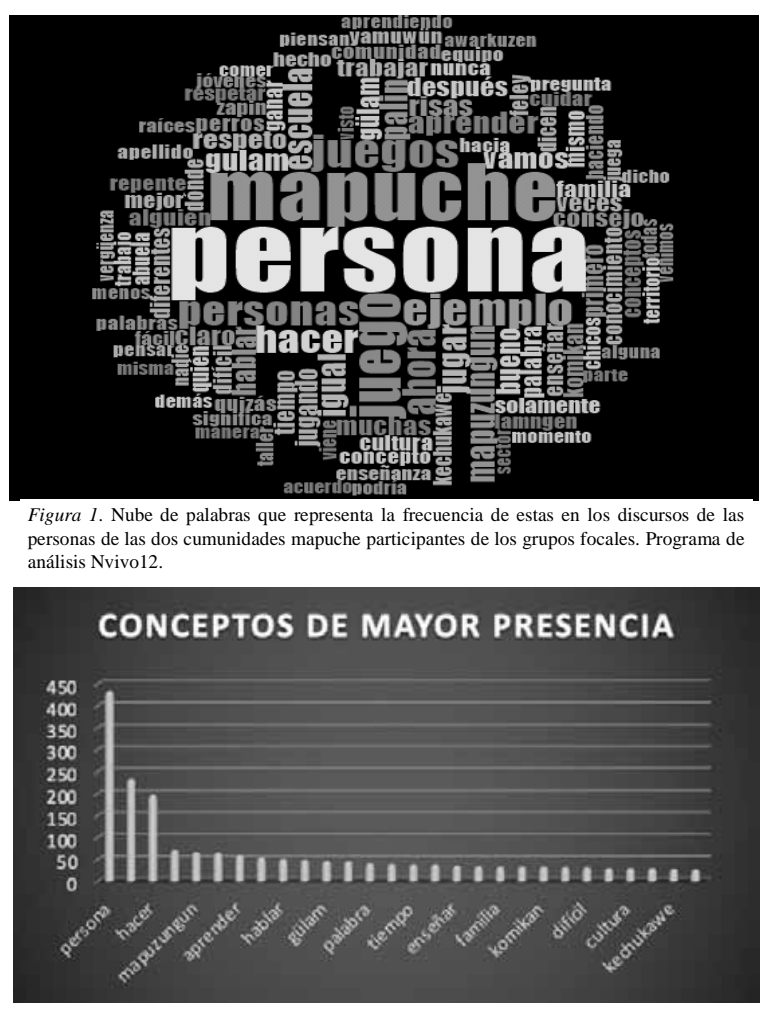

Figura 2. Gráfica de presencia de palabras mas nombradas por niños y niñas partiicpantes de los grupos focales. su hacer o más bien entendido a partir de las formas que se tiene de hacer las cosas en el contexto de la escuela, que es el espacio donde experimentaron el juego mapuche.

La frecuencia con la que aparecen las diferentes temáticas que han emergido de nuestros análisis es bastante homogénea. Sin embargo, hay tres grande ideas que poseen una presencia mucho mayor que resto, a saber: persona, mapuche y hacer.

El análisis de contenido y relacional de todas estas temáticas nos ha permitido llegar a tres grandes categorías de análisis sobre las que iremos profundizando a continuación. Las temáticas Aprender jugando, Valores del juego y Orientaciones metodológicas surgen de los comentarios tanto de las comunidades como de los niños y niñas. El caso de la temática Negación de la cultura surge exclusivamente de los focos grupales de las comunidades consultadas. La tabla $n^{0} 3$ describe la cantidad de referencias encontradas en estas temáticas.

Tabla $\mathrm{n}^{\circ} 3$.

Nodos creados a partir de la síntesis y agrupamiento de los criterios teóricos.

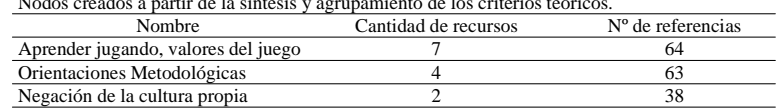

Es interesante mostrar cómo para la comunidad de Padre Las Casas, el juego es la forma en la que los niños(as) aprenden su cultura, lo que les es propio, y van desarrollando su identidad. En este proceso, el juego posee, muestra y representa diversos valores y usos (más adelante profundizaremos sobre ello). Además, los sujetos participantes son muy enfáticos en señalarnos que esos valores y usos tienen que ver con ciertas intenciones construidas desde las propias comunidades o al interior de la cultura.

Es por ello que la segunda categoría que mostramos es la de orientaciones metodológicas que posteriormente caracterizaremos. Estas son una serie de características que las comunidades en general y los educadores y niños(as) debieran consideran para hacer del juego una lógica que permita una transmisión adecuada de la cultura mapuche.

Haciendo referencia a la tercera categoría de mayor presencia en nuestro análisis, los sujetos participantes nos hacen una llamado de atención sobre el peligro, siempre presente, de negar aquello que como mapuches les es propio. Profundizan cómo la cultura occidental hace esfuerzos sistemáticos por negar aquellas formas de sentir, pensar y jugar de las comunidades indígenas del sur de Chile.

Queremos sintetizar estas ideas preliminares a partir de la figura 3.

A continuación, vamos a profundizar en la caracterización de cada una de estas categorías. Para ellos aprovecharemos el discurso textual de los sujetos participantes en los grupos focales donde ellos han participado.

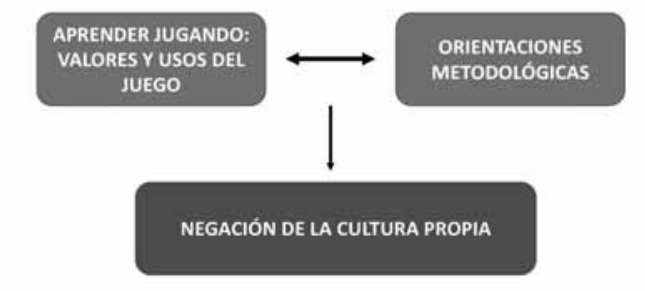

Figura ${ }^{\circ}$ 3. Conceptos teóricos emanados de la síntesis de agrupamiento temático. 


\section{Discusión de resultados}

\section{Aprender jugando: valores y usos del juego}

Un primer aspecto que llama poderosamente la atención cuando los participantes hacen referencia al juego es el entendimiento, que la competitividad entorpece y dificulta el aprendizaje de los verdaderos valores que podrían encarnarse desde el juego. Eluney ${ }^{1}$ plantea que es imprescindible «cumplir una regla general» relacionada con el «eliminar la competitividad que exista en cada juego». De lo contrario, «si es el trofeo el motivo por el que se juega, habrá competitividad y eso posee un fuerte sesgo, perdiendo el sentido y el valor del propio juego, pues se centrar en el ganar y no en el jugar».

Los/las participantes insisten en que el juego mantendrá su valor educativo cuando «no nos picamos por perder y ganar», comenta Nahuel. Yankiray es explícita al decirnos que «no hay que enojarse o picarse si uno pierde» y menos aún, comenta Aylin, «ofender al otro». Es muy interesante el recordatorio que plantea Lautaro en relación al jugar en las comunidades: «a todos nos pasan cosas mientras jugamos, somos humanos, es normal eso. Lo importante es acordarse y no picarse». Existe un claro reconocimiento de la comunidad, niños y niñas participantes mapuche acerca del sentido que la cultura mapuche da al ser persona, en este sentido el carácter simbólico que se le atribuye está relacionado con el ideario de los valore mapuche vinculados a saber relacionarse entre pares desde el respeto y el sentido de justicia y valoración, en la lengua mapuche esto sería Yamuwün en el juego.

El juego también permite construir aprendizajes importantes para la vida a través del poder responsabilizarse de ciertos compromisos de y con la comunidad. Mailen es enfática en plantear que «cuando uno es chico, generalmente a uno lo mandaban a cuidar animales y ahí es cuando uno se conoce con otros niños que cuidan animales. Y esto es como un juego». El sentido de compromiso y deber de las labores asignadas dentro de la comunidad son parte de la construcción del ser persona también, el Azmuwün se manifiesta en esta reflexión pues es en el entendimiento de las cotidianidades y la relación directa con el entorno (entorno que es contexto del juego también) donde se d aprecio a la diversidad del todo lo que nos rodea.

En los juegos experimentados, el respeto y el equipo son primordiales. Tahiel nos comparte la siguiente idea: «hay que respetarnos como somos, hay que valorar nuestras raíces, de dónde venimos, no hay que apostar cosas malas sino cosas buenas que le sirvan a ellos, incluso a la familia y siempre tener cuidado y estar en equipo, nunca separarse». Este tipo de reflexiones nos lleva a comprender que se percibe en los juegos mapuche la posibilidad de encarnar el Inatuzugun, por la importancia de valorar las raíces a las que se pertenece, pero además la idea del Zapin como capacidad colectiva de cuidar y cuidarse que se puede aprender en el juego.

El juego se constituye en un espacio y tiempo pedagógico en el que «mediante el consejo se pueden aprender muchas cosas», como nos plantea Aluwe, es un ejemplo claro de que los espacios en que le Gülam se dio, fue entendido tal cual su definición lo señala desde el mapuzugun. El Gülam como aconsejamiento está presente en todos los juegos que experimentaron. Este principio de kimeltuwün es uno de los más importantes en la práctica del juego mapuche, dado en parte por la historia de oralidad que posee esta cultura y porque las formas de entender el aprender está ligado a la capacidad de escuchar y dar Gülam.

Esta última idea la reforzamos con la opinión de Alün quien expresa: «A través del juego se puede enseñar el valor del consejo, el respeto hacia los mayores, hacia los demás, porque los niños de ahora no respetan. A través de los juegos (...) se da consejos y se aprende a escuchar. Se cuentan historias que vienen de las abuelas, se le incita al niño a preguntar sobre su historia y ahí se desarrolla la identidad». Claramente al relevar la importancia del Gülam, se desprenden los otros valores y principios del kimeltuwün.

\section{Orientaciones metodológicas}

Esta temática se enmarca en el objetivo específico de describir contextualizadamente la enseñanza del juego mapuche en la lógica del kimeltuwün. En el fondo, ¿Cómo usar el juego para el aprendizaje de valores relevantes para la propia comunidad? Las respuestas a esta pregunta, son descritas por las personas participantes de esta investigación como una serie de orientaciones metodológicas necesarias, que enunciaremos en la tabla $\mathrm{n}^{\circ} 5$ a continuación.

\begin{tabular}{|c|c|c|}
\hline Nombre & $\mathrm{N}^{\circ}$ de Recursos & $\mathrm{N}^{\circ}$ de Referencias \\
\hline Aprender jugando, valores del juego & 7 & 64 \\
\hline Competencia contraria al valor del juego & 2 & 7 \\
\hline Educación, responsabilidad y respeto & 2 & 6 \\
\hline No picarse & 2 & 5 \\
\hline Yamuwün como respeto & 1 & 5 \\
\hline Gülam como consejo & 4 & 5 \\
\hline Sapin como cuidarse & 2 & 7 \\
\hline Importancia del palin & 1 & 4 \\
\hline Azmuwün como encontrarse & 2 & 4 \\
\hline Inatuzugun como rescatar raíces & 1 & 3 \\
\hline \multicolumn{3}{|c|}{$\begin{array}{l}\text { Tabla n }{ }^{\circ} 5 . \\
\text { Principales subnodos del nodo Orientaciones metodológicas. }\end{array}$} \\
\hline \multicolumn{3}{|l|}{ Sub nodos por concepto teórico } \\
\hline Nombre & $\mathrm{N}^{\circ}$ de Recursos & $\mathrm{N}^{\circ}$ de Referencias \\
\hline Orientaciones Metodológicas & 4 & 62 \\
\hline Relevancia del contenido y la comunidad & 3 & 15 \\
\hline Importancia de agradecer & 2 & 12 \\
\hline Autonomía e identidad & 3 & 10 \\
\hline Relevancia del mapuzugun & 2 & 7 \\
\hline Interfaces cultura mapuche cultura occidental & 2 & 6 \\
\hline Mas hacer menos decir & 1 & 4 \\
\hline
\end{tabular}

\section{Relevancia del contexto y la comunidad}

Paillakewün plantea que es relevante «hacer participar y trabajar a la comunidad, pues desde ahí se puede compartir. Es una forma de conocerse y de construir una vida centrada en el compartir y no en la individualidad». Uno de los aspecto relevantes del aprender a ser persona, dentro de la cultura mapuche, lo verbalizan los participantes de esta investigación, señalando que el trabajo de la educación y en este caso el juego mapuche debe ser enseñado involucrando a las familias en los procesos, de esta forma es posible comprender el sentido de comunidad por sobre la individualidad, así como el respeto y la valoración de las personas mayores.

\section{Agradecimiento cómo lógica relacional}

El agradecimiento es una forma de la relacionarse, pero cuando los participantes mencionan este aspecto se están refiriendo en las lógicas del kimeltuwün y del mañummuwün 
en relación no solo entre personas, sino también a la relación con toda la naturaleza. Kuramil nos comenta, en este sentido que el agradecimiento «se entiende en un sentido amplio, de la naturaleza, de todo, ósea no solo de las personas». Este principio en el kimeltuwün nos encamina a entender que para aprender ser una persona agradecida es fundamental aprender de todo el entorno que rodea a los niños y niñas, pues esta es la forma en la que va adquiriendo el respeto, la valoración y la importancia que tienen las personas, el entorno y todos los seres que acompañan la vida del mapuche. En el contexto del juego recordamos que el Komikan, Kechukawe son juegos de comprensión del entorno.

\section{Autonomía e identidad como grandes propósitos del jugar}

El juego posee un carácter profundo en su intencionalidad cultural. Debe servirnos, según los participantes, para: «enseñar la historia mapuche. Historia del pueblo, del sector, de la comuna. Es muy relevante que esto se aprenda y que nos sintamos orgullosos de saber que Caupolican hizo esto, que Leftraru hizo esto otro...» (Millangürü). Esta reflexión nos refuerza las ideas del Inatuzugun, es decir la importancia de las raíces ancestrales, como dice Aneley: «aprender a reconocer nuestras raíces, de dónde venimos». El aprendizaje del origen familiar «profundizar en la sabiduría y el conocimiento. Ahí nosotros le estaríamos entregando un valor al juego» (Millangürü) y por tanto es en este sentido que el juego cumple un rol de identidad o conformación de la misma. Este proceso requiere de la utilización constante del Rakizuam (razonamiento) por parte de niños y niñas que juegan, con la guía de quien enseña, quien además debe comprender que proceso de desarrollo de la autonomía está ligada a la identidad que se forma en el juego.

\section{Enseñanza del mapuzugun}

Existe coincidencia en la propuesta metodológica que hacemos de relacionar los conceptos del kimeltuwün desde el mapuzugun, pero más específicamente los y las participantes nos hablan de materializar la lengua en toda la enseñanza, es decir enseñar el juego en mapuzugun, «un juego mapuche no está completo si no hay comunicación en Mapuzugun» (Ayinray). Este es uno de los desafíos más importantes pues en la condición actual en la que se encuentra la lengua, esta reflexión se torna central y nos hace considerar la falta de manejo del mapuzugun de educadores/as para cumplir con los objetivos centrales del aprendizaje del juego mapuche en las lógicas del kimletuwün. Refuerza esta idea Leftraru diciendo que «Ahí hay un vacío del aprender primero en Mapuzugun, para que se comuniquen mediante el juego, por ejemplo el juego, el palin hay harto concepto mapuche ahí y lo ideal sería comunicación en Mapuzugun».

A nivel del complejidad de la comunicación y del lenguaje no es suficiente aprender ciertas palabras del juego mapuche, como dice Küyenray «igual, no solamente que conozcan un concepto básico como el wechuy (se terminó), esas son simplemente palabras, si no saben el significado, si no saben él porqué». No basta manejar un par de palabras para considerar que se está enseñando juego mapuche desde el kimeltuwün, hace falta comprender las lógicas relacionales desde las formas lingüísticas e interpretativas que la cultura mapuche da a los conceptos que encarnan el juego, esta relación dialéctica del lenguaje con el juego mapuche.

Wenchumañ, nos plantea la importancia de vivir el mapuzugun para «poder entender y entender el conocimiento que se transmite a través de ese juego, para poder comprender cómo se transmiten los valores (...)». Esto nos hace considerar nuevas aristas a las críticas que los autores nos señalan en el marco teórico en los procesos de interculturalidad. El mapuzugun debe ser una competencia profesional de quien eduque en contexto mapuche (sea sus estudiantes, sea contenidos culturales) de otro modo es imposible el aprendizaje del juego en términos interculturales y menos desde las lógicas del kimeltuwün.

\section{Interfaces cultura mapuche $<>$ cultura occidental}

Es muy interesante que a pesar de todo lo dicho anteriormente, los sujetos participantes en la investigación consideran que es necesario y positivo pensar en interfaces productivas entre la cultura mapuche y la cultura occidental, viendo en la institución educativa formal un instrumento para rescatar la cultura propia. Es decir la escuela, en este contexto, es un potencial espacio de cambio paradigmático del aprendizaje mapuche más allá de la interculturalidad funcional.

Lonkotraru plantea en el «colegio se les ha enseñado bastante a los chicos, desde el jardín hasta el colegio. El jardín es una educación inicial muy fuerte, muy importante. Se desarrolla una base muy importante del mapuzugun, y eso después continúa en la escuela. Eso ha motivado mucho a la comunidad para practicar el mapuzugun» Sin embargo, atendiendo a las reflexiones anteriores, no debemos olvidar que para que la escuela tenga un protagonismo importante y significativo en los procesos de revitalización y pervivencia de la cultura mapuche esta debe atender las lógicas del kimeltuwün de la mano del mapuzugun

\section{Más hacer, menos decir}

Es importante hablar de la cultura mapuche, según los sujetos participantes, pero lo más relevante es que las instituciones educativas puedan pensar, planificar y ejecutar acciones que contribuyan a rescatar y enseñar la cultura propia de manera concreta y sostenida en el tiempo. Debe conocer y hacer parte de la educación de los niños y niñas a las familias, la comunidad completa, con sus autoridades ancestrales y las personas en general.

Aluwe, lo plantea así:

La escuela debería trabajar con la historia. La escuela, por su forma de trabajar, puede ocupar a los padres y apoderados desde sus propias intenciones, necesidades, explicaciones y aspiraciones colectivas. Ahí está el zapituwün o el zapin. Nosotros cuidamos todo lo que tiene que ver con el territorio, con el lof, y ahí entran las autoridades, futakeche fey kimel ta zugu, Lonko, machi, etc... Hablar de ello permite tener un enfoque distinto a lo que hoy día se ha estado trabajando históricamente en la escuela en relación a los juego.

\section{Negación de la cultura propia}

En este último apartado daremos cuenta de la similitud 
de la crítica que hace al marco de referencia de este artículo, acerca de las dificultades de la circulación de los saberes propios en el contexto escolar por desconocimiento y falta de intención de romper con la monoculturalidad educativa chilena (Quilaqueo, 2006; Quilaqueo y Quintriqueo, 2008; Quilaqueo y Quintriqueo, 2010 y Duran y Catriquir 2007), también lo que señalan acerca de los asistencialismos e intentos fallidos de la educación intercultural carente de mapuzugun(Quilaqueo y Torres, 2010; Poblete, 2018 y Naguil 2018) que más bien ha sido una interculturalidad funcional (Walsh, 2010) sin una crítica profunda o efectivamente mejorada. Esto es lo que dificulta los procesos de las lógicas internas dentro de la cultura mapuche, el juego como parte de la identidad cultural identitaria de lo mapuche se ve altamente alterado por la educación hegemónica impuesta. Estamos aludiendo a lo que las entrevistadas denominaron como la «negación de la cultura propia». En dicha negación aparecen algunos aspectos (ver tabla $n^{0} 6$ ) que son relevantes y que se describen a continuación.

\begin{tabular}{lcc}
$\begin{array}{l}\text { Tabla } \mathrm{n}^{\circ} 6 . \\
\text { Principales subnodos del nodo Negación de la cultura propia. }\end{array}$ & $\begin{array}{c}\mathrm{N}^{\circ} \text { de } \\
\text { recursos }\end{array}$ & $\begin{array}{c}\mathrm{N}^{\circ} \mathrm{de} \\
\text { referencias }\end{array}$ \\
\hline Nombre & 2 & 13 \\
\hline Negación de la cultura propia & 2 & 7 \\
Escuela como enculturación & 1 & 7 \\
Pérdida de identidad & 1 & 2 \\
Modernidad debilita la construcción de la identidad de la cultura & 1 & 2 \\
Libros escolares como negación de la cultura propia &
\end{tabular}

La negación de la propia cultura en la voz de las personas de la comunidad, plantea que como característica central de la experiencias vividas por ellos mismo es la fuente que desarrolla esta negación como una forma de autoprotección: «la discriminación que ha existido (...) Dicha discriminación ha generado que muchos escondan nuestra propia cultura, dificultando así jugar desde nuestros propios juegos» (Wenchuleufu).

Además, en el ámbito concreto de la educación formal, uno de los elementos relevantes en esta negación han sido los libros y los contenidos tergiversados con lo que se aprende. «Los libros son la principal traba para aprender la cultura mapuche en estos tiempos (...) (Widaleufu) «Ahí es donde un niño pierde todo el incentivo. A mí me paso eso que digo, hice mi práctica en una escuela cercana a mi casa y ahí llegaban esos libros, y yo como era hablante ayudaba a la profesora, y los chicos no entendían nada y ahí es donde dejan el libro en la mesa y ya se les olvida todo. Generalmente no traen una enseñanza que vaya acorde a la cultura, que vaya tan aferrado por ejemplo a la historia mapuche, siempre son cuentos, cosas que a uno a veces no le llaman la atención» (Widaleufu). Esta reflexión pone en evidencia el desconocimiento que profesores y profesoras tienen de sus propios estudiantes. El desafío del kimeltuwün entonces va más allá del contexto escolar local, se hace necesario permear además las instituciones formadoras de profesores, profesoras y educadores/es tanto en las lógicas mapuches sociales como el kimeltuwün y la lengua como canal de comunicación desde este saber.

\section{Conclusiones}

Finalmente señalar que el impacto sociocultural de juego mapuche para la transmisión de concomimiento al interior de la propia cultura en las lógicas del kimetuwün, es determinante para los procesos de revitalización y pervivencia, sin embargo deben existir ciertas garantías desde las posibilidades de enseñanza para que el kimeltuwün en el juego mapuche circule. Uno de ellos es la importancia del valor del juego en el contexto del respeto (yamuwün), el cuidar las actitudes competitivas e individualistas, favorecer las comunitarias y el saber escuchar y dar consejo (gülam) para generar conciencia y diálogo, el cuidado y el agradecimientos como actitud permanente en lo valórico de todo lo que nos rodea. Todo lo anteriormente dicho con el fin de formar a través del juego a personas correctas. Esto sería la misión del juego mapuche en su matriz cultural, la de formar personas con los valores propios que la cultura mapuche ha ido desarrollando a lo largo de su existencia, esto sería: ser una persona con conocimientos sólidos en su cultura y el entorno en general (kimche), ser una persona derecha en su actuar y su discurso (norche), ser una persona con una energía fuerte y poderosa para enfrentar los desafíos de la vida (newenche) y ser una persona de buenos sentimientos, sincera y leal (kumeche). Desde el punto de vista metodológico la lengua mapuche es significante y canal educativo del juego, la participación e involucramiento de la comunidad y la familia, las relaciones dialógicas desde el agradecimiento y el compromiso al derecho de la autonomía y la identidad transmitida en el juego no deben estar aislada de su planificación. Así bien, los conceptos elegidos en esta investigación desde el kimeltuwün (mañummuwün, azmuwün, gülam, inatuzugun, yamuwün y zapin) son potencialmente adaptable a las metodologías de enseñanza en contexto. Sin embargo, dichas palabras deben ser entendidas en los contextos históricos en los que circulan actualmente la nación mapuche para desde ahí comprender las formas de adaptarlas a dichos contextos para la misma gente mapuche como los otros contextos en los que se quisiera aprender en las lógicas del kimeltuwün.

\section{Agradecimientos}

Esta investigación fue financiada por el Fondo Nacional de desarrollo de las artes y la Cultura FONDART, convocatoria regional 2017, Chile. Investigación en la línea Pueblos originarios. Folio $n^{\circ} 425821$. Especial agradecimientos a las comunidades y escuelas participantes de este proceso.

\section{Referencias}

Altuve, E. (2009). Deporte: ¿Fenómeno natural y eterno o creación socio-histórica? Espacio Abierto Cuaderno Venezolano de Sociología, 18(1), 7-23.

Altuve, E. (2009). Juego y Revolución. Revista de Historia do Esporte, 2(2), 1-24.

Cagigal, J. (1976). Cultura intelectual, Cultura Física. Citius, Altius, Fortius, XVIII (1-4), 25-51.

Calvo, C. (2008). Del mapa escolar al territorio educativo. Santiago de Chile: Ed. Nueva Mirada.

Capretti, S. (2011). La cultura en juego: el deporte en la sociedad moderna y post-moderna. Trabajo y sociedad, 15(16), 231250.

De Sousa Santos, B. (2000). Crítica de la Razón indolente: Contra el desperdicio de la experiencia. Bilbao: Editorial Descleé De Brouwer. 
Duran, T., y Catriquir, D. (2007). Interculturalidad en la vida académico-social: un desafío intereepistemológico. Patrimonio cultural mapunche. Temuco: Universidad católica de Temuco.

Edwards, D., \& Stokoe, E. (2004). Discursive psychology, focus group interviews and participants categories. British Journal of Developmental Psychology, 22, 499-507. Doi: 10.1348/ 0261510042378209

Fairclough, N. (1995). Critical Discourse Analysis: the Critical Study of Language. London: Longman.

Gallego-Ortega, J.L., y Rodríguez-Fuentes, A. (2018). Percepciones del profesorado sobre competencias comunicativas de futuros maestros de educación física. Revista Internacional de Medicina y Ciencias de la Actividad Física y el Deporte, 18(71), 479-492. Doi: 10.15366/rimcafd2018.71.005

Gonçalves, L., Aparecida, D., y Rodríguez, J. (2018). Vivencia del ocio entre hombres: los juegos de bocha (São Paulo, Brasil) y bolos tradicionales (Boiro, España). Revista Retos. Nuevas tendencias en Educación Física, Deportes y Recreación, 33, 129133.

Hopenhayn, M. (2005). América Latina desigual y descentrada. Buenos Aires: Grupo Editorial Norma.

Huizinga, J. (1949). Homo Ludens, A study of the play-element in cultura. London: Routledge.

Kusch, R. (1977). Pensamiento popular e indígena en América. Buenos Aires: Hachette.

Loncon, E. (1997). Construyendo una educación intercultural bilingüe mapuche. Propuestas y desafíos. Temuco: CONADI, SIEDES.

López, C. (2011). El Palín, juego tradicional de la cultura Mapuche. Valparaíso: Ediciones Universitarias de Valparaíso.

López, C. (2013). Pillmatun y Linao: dos juegos de pelota de manos originarios de la cultura mapuche. Valparaíso: Ediciones Universitaria de Valparaíso.

Manquilef, M. (1914). Comentarios del pueblo Araucano (juegos, ejercicios y bailes). Anales de la Universidad, CXXXIV, 1-60.

Miles, M. B., \& Huberman, A. M. (1994). Qualitative data análisis: an expanded sorcebook. Calfornia: Sage Publications.

Moor, P. (1987). El juego en la educación. Madrid: HERDER.

Morse, J. M. (2005). Asuntos críticos en los métodos de investigación cualitativa. Alicante: Universidad de Alicante.

Paillalef, J. (2003). Los mapuches y el proceso que los convirtió en indios. Psicología de la discriminación. Santiago de Chile: Universidad Tecnológica Metropolitana.

Poblete, C. (2018). Kom kake az-mongen wallontu mapu mew nieyngün ñi awkantun, pu Mapuche ka RapaNui ka fey5: Análisis crítico a las iniciativas del Ministerio del Deporte en Chile para su revitalización. En M. Herrera., D. Hincapié. Y W. Moreno (Eds.), Juegos y Deportes Autóctonos, Tradicionales y Populares. (pp. 78-91). Madrid: Editorial Académica Española.

Quidel, J. (2002). Orientación para la incorporación del conocimiento mapuche al trabajo escolar. Gobierno de Chile: Ministerio de Educación.

Quilaqueo, D. (1996). Construyendo Interculturalidad. Experiencia de Formación Docente en Educación Intercultural Bilingüe. En J. Jolibert, I. Cabrera, G. Inostroza y X. Riveros (Edss.). Transformación de la formación docente inicial. Propuesta e didáctica de la lengua materna. (pp. 201-217). Santiago de Chile: UNESCO.

Quilaqueo, D. (2005). Educación Intercultural desde la Teoría del Control Cultural en Contexto de Diversidad Sociocultural Mapuche. Cuadernos Interculturales, 3(4), 37-50.

Quilaqueo, D. (2006). Valores educativos mapuches para la formación de persona desde el discurso de kimches. Estudios Pedagógicos, 32(2), 73-86.
Quilaqueo, D., y Quintriqueo, S. (2010). Saberes educativos mapuches: un análisis desde la perspectiva de los kimche. Polis, 9(26), 337-360.

Quilaqueo, D. y M. Merino (2003). Estereotipos y prejuicio étnico hacia los mapuches en textos complementarios a la asignatura de Historia. Revista Campo Abierto, 23, 119-135.

Quilaqueo, D., Quintriqueo, S., Catriquir, D., y Llanquinao, G. (2003). Kimeltuwün mew amukey ta zugu: una didáctica para abordar conocimientos mapunche en el proceso de formación inicial en educación intercultural. En AA.VV. Cuatro estudios para mejorar la formación inicial docente. (pp. 57-148). Temuco: Universidad Católica de Temuco.

Quilaqueo, D. (2006). Valores educativos mapuches para la formación de persona desde el discurso de kimches. Estudios pedagógicos, 32(2), 73-86. Doi: 10.4067/S071807052006000200004

Quilaqueo, D., y Torres, H. (2013). Multiculturalidad e interculturalidad: desafíos epistemológicos de la escolarización desarrollada en contextos indígenas. Alpha, 37, 285-300. Doi: 10.4067/S0718-22012013000200020

Quintriqueo, S., y Maheux, G. (2004). Exploración del conocimiento sobre la relación de parentesco como contenido educativo para un currículum escolar intercultural en comunidades mapuche. Revista de Psicología, XIII (1), 73-91.

Rodríguez, G., Gil, J., y García, E. (1999). Metodología de la investigación cualitativa. Málaga: Aljibe.

Rodríguez, J., Oliveira, B., y Navarro, R. (2019). Análisis de las prácticas lúdicas y tradicionales en la en la zona norte del Camino Central Portugués y su relación con el ámbito educativo. Retos. Nuevas tendencias en Educación Física, Deportes y Recreación, 35, 25-30.

Strauss, A., \& Corbin, J. (2002). Bases de la investigación cualitativa: Técnicas y procedimientos para desarrollar la teoría fundamentada. Medellín: Universidad de Antioquia.

Toldi, S., Fiorito, M. E., y Álvarez, M. (2006). Grupo focal y desarrollo local: aportes para una articulación teóricometodológica. Revista Ciencia, Docencia y Tecnología, XVII(033), 111-131.

Torres, J. (2001). Educación en tiempos de neoliberalismo. Madrid: Ed. Morata.

Valles, M. (1998). Técnicas cualitativas de investigación social. Reflexión metodológica y práctica profesional. Madrid: Editorial Síntesis.

Van Dijk, T. (2003). El discurso como estructura y proceso. Barcelona: Editorial Gedisa.

Walsh, C. (2009). Interculturalidad Crítica y educación intercultural. Ponencia presentada en Seminario Interculturalidad y Educación Intercultural, La Paz: Instituto Internacional de Integración del Convenio Andrés Bello. Recuperado de http:// w w w. u c h i l e.c l/d o c u m e n t o s / interculturalidadcriticayeducacionintercultural_110597_0_2405.pdf

Wodak, R. (2003). De qué trata el análisis crítico del discurso. Resumen de su historia, sus conceptos fundamentales y sus desarrollos. En Wodak, R., y Meyer, M. (Eds.). Métodos de análisis crítico del discurso. (pp. 17-34). Barcelona: Editorial Gedisa.

\section{(Footnotes)}

${ }^{1}$ Todos los nombres que en este informe aparecen son ficticios. Ello con la intención de resguardar la identidad de los participantes. 